\title{
Molecular Characterization of Nosocomial Methicillin Resistant Staphylococci aureus by Rep-PCR
}

\author{
S Nordin, Z Wahab, S Hussin, M Rahman
}

\begin{abstract}
Citation
S Nordin, Z Wahab, S Hussin, M Rahman. Molecular Characterization of Nosocomial Methicillin Resistant Staphylococci aureus by Rep-PCR. The Internet Journal of Infectious Diseases. 2010 Volume 9 Number 2.
\end{abstract}

\begin{abstract}
Nosocomial methicillin-resistance Staphylococcus aureus (MRSA) poses problem to clinicians and hospital administrators for its management. In the present study isolation, identification molecular characterizations of methicillin-resistance Staphylococcus aureus were performed at Hospital Kuala Lumpur, Malaysia during December 2005 to April 2007. Twenty seven MRSA positive samples were identified based on cultural, biochemical and antibiotic sensitivity assay. These were, $51 \%$ from blood samples, 33.3\% from tracheal aspirate and 11,1\% from nasal swab and pus. Molecular method rep-PCR was used to characterize the MRSA positive strains. Results of rep-PCR showed 5 different pattern of bands based on their genomic nature. Thus, rep-PCR was proved to be potential tool to determine genomic differences of nosocomial MRSA in resource limited setting.
\end{abstract}

\section{INTRODUCTION}

Staphylococcus aureus is considered as a major nosocomial pathogen which causes a range of diseases, including endocarditis, osteomyelitis, pneumonia, toxic-shock syndrome, food poisoning, carbuncles, and boils.

Infection with nosocomial methicillin-resistance Staphylococcus aureus (MRSA) in hospitals poses significant problems to clinicians managing patients, as well as to hospital administration. Since the introduction of methicillin in the 1960s, prevalence of nosocomial MRSA infections increased globally. Many countries have reported periodic outbreaks of nosocomial infections with MRSA especially in the intensive care units. In the United States, the National Nosocomial Infection Surveillance System reported the increase of MRSA in the intensive care units from 3\% to $53 \%$ over 20 years. In Malaysian hospitals the rate of MRSA in 1988 reported to be between $10-25 \%{ }^{3}$ Further data obtained from the National Surveillance on Antibiotic Resistance program showed increase in the percentages of MRSA from 28\% in 1992 to 36\% in 1996. However, in the past four years, the rates of MRSA infections in 17 major hospitals showed decreased in trend.

Detection and characterization MRSA strains during outbreaks are important to monitor trace and control the intrahospital and interhospital spread or evolution of bacterial strains. Molecular methods have been proven to be cost-effective for the study of nosocomial outbreaks and give genomic nature of infected organisms. However, PFGE is time consuming, tedious and the reagents are expensive, hence may not be practical in resource limited settings. RepPCR seems to be a promising technique in view of its adequate discriminatory power, good reproducibility and at a low cost in detecting MRSA. ${ }^{5,6}$ The present communication deals with use of rep-PCR to characterize genetic relatedness among the MRSA strains isolated and identified from clinical samples.

\section{MATERIALS AND METHODS STUDY PLACE}

The present research was conducted on the patients admitted to Hospital Kuala Lumpur (HKL), Malaysia from December 2005 to April, 2007.

\section{SPECIMENS}

Blood, tracheal aspirate, nasal swab and pus specimens obtained from the suspected patients were processed in the Microbiology laboratory for the isolation and identification of Methicillin Resistant Staphylococcus aureus(MRSA) organisms.

\section{ISOLATION AND IDENTIFICATION}

Methicillin Resiatant Staphylococcus aureus(MRSA) organisms were isolated and identified based on colonial morphology, Gram's stain, Coagulase test, Antibiotic resistance to methicillin by oxacillin $1 \mu \mathrm{g}$, disk susceptibility 
test following the guidelines of the Clinical and Laboratory Standards Institute. ${ }^{7}$

The MRSA identified bacteria were kept in microbank bead and stored at $-70^{\circ} \mathrm{C}$. These were sub-cultured on blood agar before use.

\section{ANTIBIOTIC SENSITIVITY TEST}

Antibiotic susceptibility tests were performed by disc diffusion method using the Mueller-Hinton agar which was inoculated with a suspension adjusted to $0.5 \mathrm{McFarland}$ turbidity standards. Plates were incubated overnight at $35^{\circ} \mathrm{C}$. CLSI guidelines were used as reference for determination of susceptibility. In addition to oxacillin $(1 \mu \mathrm{g})$, the antimicrobial panel include fusidic acid $(10 \mu \mathrm{g})$, mupirocin $(5 \mu \mathrm{g})$, clindamycin $(2 \mu \mathrm{g})$, vancomycin $(30 \mu \mathrm{g})$ gentamicin (10 $\mu \mathrm{g})$, erythromycin $(15 \mu \mathrm{g})$, cotrimoxazole (1.25 / 23.75 $\mu \mathrm{g})$, rifampicin $(5 \mu \mathrm{g})$, linezolid (30 $\mu \mathrm{g})$ and teicoplanin (30 $\mu \mathrm{g})$. Reading of the inhibition zone and interpreting susceptibility were performed using the Biomic Vision Microbiology Analyser.

Quality control strains methicillin-sensitive Staphylococcus aureus ATCC 25923 was included in the testing. Confirmatory test for MRSA detection was confirmed by performing PCR to detect mecA gene, adapted from study done by Delvecchio et al. ${ }^{8}$

\section{PATIENTS' PROFILES}

Patients' profiles were retrieved from the case files of patients with the isolated MRSA, from the medical record office.

\section{MOLECULAR METHOD}

DNA extraction was performed by High Pure PCR Template Preparation Kit (Roche Diagnostics, Germany). The nucleotide sequences of the primers to amplify targets in the rep-PCR of MRSA were obtained from previously published article by Delvecchio et al. ${ }^{8}$ Briefly $25 \mu \mathrm{L}$ of Master Mix (Roche Diagnostics, Germany) in which there was $1 \mu \mathrm{L}$ of primer, $23 \mu \mathrm{L}$ of sterile double distilled water and $1 \mu \mathrm{L}$ of DNA. The thermo-cycling conditions were: initial denaturation at $95{ }^{\circ} \mathrm{C}$ for 3 minutes, denaturation at $94{ }^{\circ} \mathrm{C}$ for 1 minute, annealing at $54{ }^{\circ} \mathrm{C}$ for 1 minute and extension at $72{ }^{\circ} \mathrm{C}$ for 2 minutes. Thirty cycles were performed followed by a second extension at $72{ }^{\circ} \mathrm{C}$ for 5 minutes. In every PCR run, a negative control (nuclease free water) was included. Methicillin-sensitive Staphylococcus aureus was included as control strain when running the rep-PCR. A $5 \mu \mathrm{L}$ of reaction product was added to $1 \mu \mathrm{L}$ of loading dye and were electrophoresed on the $1.5 \%$ composite agarose gel (Promega, USA, catalogue number V3121) in Ultra PureTM TBE Buffer (Promega, USA) at 90 volt for 30 minutes. Visualisation of the resulting DNA bands was accomplished by ethidium bromide staining and image was captured under ultraviolet reader using Gel Doc by Biorad Corporation Software.

\section{ETHICAL CONSIDERATION}

The study was approved by the Research and Ethical Committee of Medical Faculty, UKM

\section{RESULTS}

\section{ISOLATION AND IDENTIFICATION OF BACTERIA}

It was observed that twenty seven samples were positive for MRSA obtained from 23 patients (4 patients had two MRSA isolates), who were admitted to Hospital Kuala Lumpur. 14 (51.9\%) of the MRSA isolates from blood samples, 9 (33.3\%) from tracheal aspirate, $3(11.1 \%)$ from swab samples, 2 of which were nasal swabs and 1 (3.7\%) was pus.

\section{FINGERPRINTING PATTERNS OF MRSA}

Methicillin-sensitive Staphylococcus aureus was included as control strain when running the rep-PCR. The patterns of bands from the MRSA isolates vary with 4 to 5 bands visible, ranging in size from $210 \mathrm{bp}$ to $1150 \mathrm{bp}$ (Fig 1). As shown in the figure-1, the MSSA patterns were different from that of MRSA, where MSSA isolates lack the $650 \mathrm{bp}$ band that was seen in the MRSA isolates. The rep-PCR produced variable patterns on the agarose gel and could differentiate the isolates into five groups, designated as ' $a$ ' to ' $\mathrm{e}$ '. In this study MRSA strain belonging to PCR pattern 'e' is the predominant strain his strain was present throughout the study period, in December 2005, then November 2006 till April 2007. It is interesting to see that patient ' $\mathrm{B}$ ', with repeated blood samples sent 3 days apart, and MRSA was isolated in both samples; genotypically they were not identical, hence belonged to the different strains. Likewise, two blood samples sent 2 days apart from patient ' $G$ ' did not isolate identical MRSA strains. Patient ' $\mathrm{V}$ ' had two tracheal aspirate samples sent on the same day and showed two identical MRSA strains. Patient ' $\mathrm{P}$ ' had MRSA isolated from nasal swab in January 2007, hence patient 'P' was colonized by MRSA. In April 2007, MRSA was isolated from patient 'P's blood sample and this strain were identical to the colonizer isolated in January 2007. 


\section{ANTIBIOGRAM ANALYSIS}

Antibiogram analysis of the MRSA collection resolved these strains into six groups designated (I-VI) (Table 1). Group II, which was resistant to cotrimoxazole, gentamicin, clindamycin and erythromycin, was the commonest accounting for $40.7 \%$ (11/27) of the isolates collected. Group I accounts for $33.3 \%$ (9/27) of the isolates and this group was resistant to cotrimoxazole, gentamicin and erythromycin. Group III accounts for $3.7 \%(1 / 27)$ of the isolates and this group was resistant to gentamicin,

clindamycin and erythromycin. Group IV accounts for $3.7 \%$ (1) of the isolates and this group was resistant to fusidic acid, cotrimoxazole, rifampicin, gentamicin and erythromycin. Group V accounts for $11.1 \%$ (3/27) of the isolates and this group was resistant to fusidic acid, cotrimoxazole, rifampicin, gentamicin, clindamycin and erythromycin. Group VI accounts for 7.4\% (2/27) of the isolates and this group was resistant to rifampicin, gentamicin, clindamycin and erythromycin.

\section{Figure 1}

Fig 1: rep-PCR generated DNA patterns in agarose gel electrphoresis.

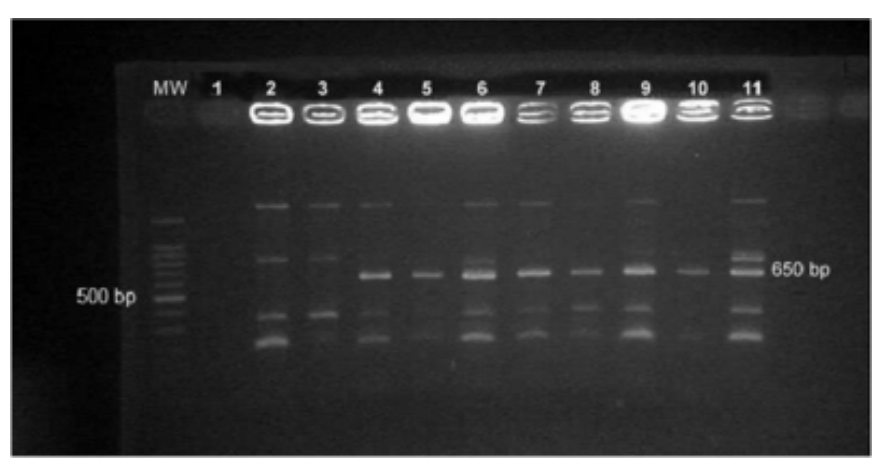

Legends

Band size: 210 to $1150 \mathrm{bp}$

Lane 1: Negative control, Lanes 2 and 3: methicillinsensitive Staphylococcus aureus isolates, lanes 4 to 11 :

MRSA :lanes 4, 7 and 9 pattern 'a'; lanes 5 and 10: pattern 'b'; lane 6: pattern 'c'; lane 8: pattern 'd'; and lane 11: pattern 'e')

\section{Figure 2}

Table 1: Antibiotic resistance and anti-biogram type of MRSA isolates

\begin{tabular}{|l|c|l|c|}
\hline \multicolumn{1}{|c|}{ Sample type } & Patient & Antibiotic Resistance & Antibiogram \\
\hline Blood & A & SXT,GM, ER & I \\
\hline Blood & E & SXT,GM,CLI,ER & II \\
\hline Blood & C & SXT,GM,ER & I \\
\hline Blood & B* & SXT, CM, ER & I \\
\hline Blood & F & SXT, GM, ER & I \\
\hline Blood & B $^{*}$ & SXT, GM, ER & I \\
\hline Tracheal aspirate & N & SXT,GM, CLI, ER & II \\
\hline Blood & M & SXT,GM, CLI, ER & II \\
\hline Blood & K & SXT,GM, CLI, ER & II \\
\hline Blood & G* & SXT,GM, CLI, ER & II \\
\hline Tracheal aspirate & N & SXT,GM, CLI, ER & II \\
\hline Tracheal aspirate & O & FU,SXT, RIF, GM, ER & IV \\
\hline Tracheal aspirate & L & GM, CLI, ER & III \\
\hline Nasal swab & P* & SXT,GM, CLI, ER & II \\
\hline Swab & R & SXT, GM, ER & I \\
\hline Blood & P* & SXT, GM, CLI, ER & II \\
\hline Pus & J & SXT,GM, CLI, ER & II \\
\hline Tracheal aspirate & Q & FU,SXT,RIF, GM, CLI, ER & V \\
\hline Tracheal aspirate & S & SXT,CM, ER & I \\
\hline Blood & G* & SXT,GM, CLI, ER & II \\
\hline Tracheal aspirate & I & SXT,GM, CLI, ER & II \\
\hline Nasal swab & H & FU,SXT,RIF, GM, CLI, ER & V \\
\hline Tracheal aspirate & V* & GM, RIF, CLI, ER & VI \\
\hline Tracheal aspirate & V* & GM, RIF, CLI, ER & VI \\
\hline Blood & W & SXT, GM, ER & I \\
\hline Blood & U & SXT, GM, ER & I \\
\hline Blood & FU,SXT,RIF, GM, CLI, ER & V \\
\hline
\end{tabular}

Legends

FU fusidic acid; SXT cotrimoxazole; GM gentamicin; RIF rifampicin; CLI clindamycin; ER erythromycin;

* Repeated samples of MRSA isolation.

\section{DISCUSSION}

This study was undertaken with a view to identification of MRSA and to do their molecular characterization which is important to monitor, trace and control of the intrahospital and interhospital spread of MRSA strains. In the present study rep- PCR was used as a tool to perform molecular study with the advantages of being simpler and less time consuming. ${ }^{9}$

In the present study 17 positive MRSA positive isolates obtained from 23 patients (4 patients had two MRSA isolates), from the patients who were admitted to Hospital Kuala Lumpur. 14 (51.9\%) of the MRSA isolates from blood samples, 9 (33.3\%) from tracheal aspirate, $3(11.1 \%)$ from swab samples, 2 of which were nasal swabs and 1 (3.7\%) was pus. 
In the study designed published primer of Delvecchio's study $^{8}$ was used to perform rep-PCR to determine the molecular nature of the above MRSA isolates.

The patterns of bands from the MRSA isolates varied with 4 to 5 bands visible, ranging in size from $210 \mathrm{bp}$ to $1150 \mathrm{bp}$ (Fig 1). By comparing the banding patterns between MSSA and MRSA, distinction between these organisms is easily determined. The MSSA isolates lack the $650 \mathrm{bp}$ band that was observed in all the MRSA isolates. The MRSA strain belonging to PCR pattern ' $\mathrm{e}$ ' is the predominant strain. This strain was present throughout the study period, in December 2005, then November 2006 till April 2007. Norazah et al. ${ }^{10}$ also determined a predominant MRSA strain in their study. It is also interesting to note in this study that patient ' $\mathrm{B}$ ', whom had repeated blood samples sent 3 days apart, and MRSA were isolated in both samples; genotypically they were not identical, hence belonged to the different strains. Therefore, patient ' $\mathrm{B}$ ' was infected by two strains of MRSA. Likewise, two blood samples sent 2 days apart from patient ' $G$ ' did not isolate identical MRSA strains. Patient ' $V$ ' had two tracheal aspirate samples sent on the same day and showed two identical MRSA strains. This makes sense since the sample was taken from the same site on the same day. Patient 'P' 's nasal was colonized by MRSA in January 2007. MRSA isolated from patient 'P's blood sample in April 2007 and this strain was identical to the colonizer isolated in January 2007. This highlights the importance of screening for MRSA carrier as an infection control measure and the role of rep-PCR in determining and verifying the MRSA strains that cause infection in a colonized patient.

There were clusters of MRSA infections observed in this study. The predominant MRSA strain in this study belonged to pattern ' $\mathrm{e}$ '. This information can be used by an Infectious Disease Physician or a Clinical Microbiologist to trace the source of the MRSA strain. Screening the environment and personnel in the ICU may show the relatedness of the MRSA between them and isolates from patients. Thus, infection control measures can then be applied to contain further spread. Van der Zee et al $^{9}$ compared repetitive element sequence-based PCR (rep-PCR), with other genotypic methods and found that rep-PCR was better than others in terns of its efficacy. They also mentioned that rep-PCR was easier and faster with good reproducibility. Delvecchio et al. ${ }^{8}$ also employed rep-PCR in their study and found similar discriminatory power.

It is also verified in this study that MRSA isolates with the same rep-PCR pattern exhibited different antibiotic susceptibility patterns, while strains with different rep-PCR patterns had the same antibiotic susceptibility. Therefore, there was no relationship between antibiotic susceptibility and rep-PCR patterns. This is in agreement with other studies that reported antibiotic susceptibility testing has a relatively limited use in molecular studies due to phenotypic variation of the isolates. ${ }^{9}$

In conclusion, genotypic method is more reliable method while performing molecular characterization and molecular epidemiology study of nosocomial MRSA pathogens. In resource-limited settings, rep-PCR has the potential implication in molecular epidemiology investigation of nosocomial pathogens to monitor, tracing and control the spread or evolution of bacterial strains.

\section{ACKNOWLEDGEMENT}

Authors acknowledge the authority of University Kebangsaan Malaysia for providing fund (FF-200-2006) to conduct the present research. The authors also acknowledge the authority of Hospital Kuala Lumpur, Malaysia for cordial assistance during the study.

\section{References}

1. Witte W, Cuny C, Klare I, Nubel U, Strommenger B, Werner G. Emergence and spread of antibiotic-resistant Gram positive bacterial pathogens. Int J Med Microbiol. 2008; 298:365-77.

2. Salgado CD, Grady NO \& Farr BM. Prevention and control of antimicrobial-resistant infections in intensive care patients. Crit Care Med 2005;33(10):2373-82.

3. Norazah A, Liew SM, Kamel AGM, Koh YT, Lim VKE. DNA fingerprinting of methicillin-resistant Staphylococcus aureus by pulsed-field gel electrophoresis (PFGE): comparison of strains from 2 Malaysian hospitals. Singapore Med J 2001; 42(1):15-19.

4. Lessing MPA, Jorden JZ \& Bowler ICJ. Molecular epidemiology of a multiple strain outbreak of methicillin resistant Staphylococcus aureus amongst patients and staff. J Hosp Infect. 1995;31:253-260

5. Olive DM \& Bean P. Principles and applications of methods for DNA- based typing of microbial organisms. J Clin Microbiol 1999; 37(6):1661-69

6. Trindade PA, McCulloch JA, Oliveira GA \& Mamizuka

EM. Braz J Infect Dis 2003; 7:32-40

7. Clinical and Laboratory Standards Institute. Performance

Standards for Antimicrobial Susceptibility Testing; Fifteenth Informational Supplement 2005; 25(1):46-47

8. Delvecchio VG, Petroziello JM, Gress MJ, et al.

Molecular genotyping of methicillin-resistant

Staphylococcus aureus via fluorophore-enhanced repetitive sequence PCR. J Clin Microbiol 1995. ;33(8):2141-2144.

9. Van der Zee A, Verbake Hl, van Zon, JC, et al. Molecular genotyping of Staphylococcus aureus strains : comparison of repetitive element sequence based-PCR with various typing methods and isolation of a novel epidemicity marker. J Clin Microbiol $1999 ; 37(2): 342-349$.

10. Norazah A, Lim VKE, Rohani MY, Alfizah H, Koh YT 
\& Kamel AGM. A major methicillin-resistant

Staphylococcus aureus clone predominates in Malaysian hospitals. Epidemiol Infect 2003;130: 407-11. 


\section{Author Information}

Syafinaz Amin Nordin

Department of Medical Microbiology and Parasitology, Putra University

\section{Zubaidah Abdul Wahab}

Department of Pathology, Sungai Buloh Hospital

\section{S. Hussin}

Department of Medical Microbiology and Immunology, University Kebangsaan

\section{M. Rahman}

Department of Medical Microbiology and Immunology, University Kebangsaan 